\title{
Multiple Hypothesis Tracking of Clusters of People
}

\author{
Manuel Mucientes \\ Department of Electronics and Computer Science \\ University of Santiago de Compostela \\ 15782 Santiago de Compostela, Spain \\ Email: manuel@dec.usc.es
}

\author{
Wolfram Burgard \\ Department of Computer Science \\ University of Freiburg \\ 79110 Freiburg, Germany \\ Email: burgard@informatik.uni-freiburg.de
}

\begin{abstract}
Mobile robots operating in populated environments typically can improve their service and navigation behavior when they know where people are in their vicinity and in which direction they are heading. In this paper we present an algorithm for tracking clusters of people using Multiple Hypothesis Tracking (MHT). The motivation for our approach is that tracking clusters of objects instead of the individual objects enhances the reliability and robustness of the tracking especially when the objects move in groups. To efficiently keep track of multiple objects and clusters, our approach uses MHT in combination with Murty's algorithm. The set of hypothesis for each iteration is constructed in two consecutive steps: one for solving the data association problem, taking also into account the frequent occlusions between the objects, and the second one for considering the joining of different clusters. Our approach has been implemented and tested on a real robot and in a typical hallway environment. Experimental results demonstrate that our approach can robustly deal with several groups of people and is able to reliably manage the splits and joins of clusters.
\end{abstract}

\section{INTRODUCTION}

The presence of people and other moving objects in the environments in which robots develop their tasks affects several fields in robotics: mapping in dynamic environments, planning, human-robot interaction, etc, and also can provide the robot with interesting information about the environment.

Many papers have dealt with the problem of moving objects tracking [1], [2]. One of the most popular approaches is Multiple Hypothesis Tracking (MHT). It was proposed by Reid [3], and it has been applied in several fields: in [4], MHT is used for visual tracking of corners, while in [5] it is implemented for tracking other robots.

An special case of tracking is that in which the moving objects are people [6], [7]. In nearly all of the approaches, individual persons are tracked. In [8], they have represented the movement of a person with a dynamical model that takes into account physical assumptions about the legs. In [9] a Samplebased Joint Probabilistic Data Association Filter has been used, showing a robust track of single people.

But all the proposed systems do the tracking for individual objects. Clustering or grouping is only mentioned in [3], but with a different purpose. The tracked objects are clustered when they compete for the same measurements, and splitted otherwise. The objective is to reduce the computation time when solving the data association. In our approach, clusters have a very different meaning. They are groups of tracks that have similar states, but tracks inside a cluster can compete for different measurements, and tracks from different clusters can compete for the same measurements. As an example, in a group of three people that walk together, the people in both extremes of the group, generally, do not compete for the same measurements, while one of these persons can compete for a measurement with a person that passes besides the group.

In real environments, usually people move in groups. As compared with the tracking of single people, the tracking of clusters of people presents a higher difficulty in the data association, not only because the tracks in a group are quite close to each other, but also because the occlusions are very frequent and not easy to predict.

To solve these problems, existing methods based on tracking each moving object individually are not appropriate. The novelty of our proposal is based in the tracking of clusters of moving objects. Each cluster has its own individual tracks (of the moving objects), but the reliability and robustness of the system significantly increases, as a more complex and close to the reality probability density function (PDF) can be constructed. In this way, the high complexity in the data association due to the existence of many close measurements and the frequent occlusions, can be managed.

The system has been implemented with the MHT algorithm [3] together with the Murty's algorithm [10] to make it computationally tractable. The main stages of the system are the data association (which measurements belong to which clusters) and the clusters join (clusters with similar states are joined). The paper is structured as follows: Section II briefly introduces the MHT algorithm, Section III describes our proposal for tracking clusters of people, Section IV presents the experimental results, while Section $\mathrm{V}$ points out the conclusions.

\section{Multiple Hypothesis Tracking (MHT)}

The MHT algorithm was proposed by Reid [3] for tracking multiple targets in a cluttered environment. As pointed out in [4], MHT integrates all the capabilities necessary to model the tracking of multiple targets:

- Creation of new tracks when new measurements appear.

- Explicit modelling of the appearance of spurious (false positive) measurements.

- Tracking of targets in the absence of measurements due to false negatives (failures in the detection) or due to the frequent occlusions among the different tracks. 
- Explicit modelling of track deletion when the target being tracked is out of the scope of the sensors, or when it has not been seen for a long period of time.

- Explicit modelling of uniqueness constraints, as we want that a measurement is assigned only to one track and vice versa in each iteration.

The MHT algorithm begins with the set of hypothesis of the previous iteration, $\Omega^{t-1}$, also called parent hypothesis set, and the set of measurements from the beginning until that iteration, $Z^{t-1}=\{Z(0), \ldots, Z(t-1)\}$. Each hypothesis $\Omega_{p}^{t-1}$ represents a different set of assignments of the set of measurements to the different tracks. Taking into account the new set of measurements, $Z(t)$, and one of the previous hypothesis, $\Omega_{p}^{t-1}$, a new hypothesis is generated, $\Omega_{k}^{t}$, making an specific assignment of the current measurements. This assignment is represented by $\psi_{p}^{t}(k)$, where $k$ is the index of the new hypothesis, and $p$ is the index of the parent hypothesis:

$$
\Omega_{k}^{t}=\left\{\psi_{p}^{t}(k), \Omega_{p}^{t-1}\right\}
$$

The set of plausible assignments, $\psi_{p}^{t}$ that can be done for a parent hypothesis $\Omega_{p}^{t-1}$ is named ambiguity matrix (sometimes also called hypothesis matrix). Each element of the matrix, $a_{i j}$, can take a value of 1 or 0 , representing the possibility that measurement $i$ is associated to a previous track, a new track, is considered noise, etc, or not. Associated to the ambiguity matrix, a cost matrix $\zeta_{p}^{t}$ must also be defined. Each element of the matrix, $c_{i j}$, represents the probability that measurement $i$ has been originated due to $j$.

From a computational point of view, evaluating all the possible assignments for all the existing parent hypothesis is intractable (NP-hard). The solution is to apply an algorithm proposed by Murty [10] to obtain the $k$-best assignments in polynomial time. This algorithm has been modified by Cox and Miller [11] in order to solve multiple assignment problems at the same time, and also to change the termination condition, as hypothesis with a likelihood below a certain percentage of the best hypothesis can be discarded.

\section{TRACKING CLUSTERS OF PEOPLE}

When people move in groups the data association gets much more complex as compared with the track of a single person. The reason is double: inside a group, the measurements are usually very close, so the possibility of making wrong data associations highly increases. But also, because of the high concentration of measurements, the frequency of the occlusions is also very high. As a consequence, the data association problem becomes really hard to solve.

For this reason, as will be pointed out in Section IV, solving the problem trying to track the moving objects individually has as a consequence very unreliable tracks. The originality of our proposal is that, instead of tracking individual objects, tracking is done using clusters. Inside the cluster, tracks can be generated or deleted in a more dynamical way, as the impact in the state of the cluster is low. The state of the cluster remains reliable and its estimations are robust, as they are based on the existing tracks (those that have high probabilities).

\section{A. Tracks and clusters}

A track is defined as a sequence of measurements along time that are assumed to belong to the same moving object. On the other hand, a cluster is a set of tracks that move together, i.e., that have similar states. The states of both the tracks and the clusters, can be represented by quadruples $\langle x, y, \theta, v\rangle$.

The prediction and update of the tracks, and the prediction of the clusters is done with an Extended Kalman Filter. To update the state of a track with a measurement $z_{m}^{t}$ it must pass the Mahalanobis distance test:

$$
\left(z_{m}^{t}-h\left(\bar{\mu}_{t}\right)\right)^{T}\left(H_{t} \bar{\Sigma}_{t} H_{t}^{T}+Q_{t}\right)^{-1}\left(z_{m}^{t}-h\left(\bar{\mu}_{t}\right)\right) \leq \eta^{2}
$$

where $z_{m}^{t}$ is measurement $m$ at time $t, h\left(\bar{\mu}_{t}\right)$ is the prediction of the measurement for the track with predicted state $\bar{\mu}_{t}, H_{t}$ is the jacobian matrix of $h, \bar{\Sigma}_{t}$ is the prediction about the state covariance, and $Q_{t}$ is the covariance of the measurement noise, while $\eta^{2}$ is the maximum allowed Mahalanobis distance.

Finally, the update of the clusters is modelled as the average of the PDFs of the tracks belonging to it.

The system designed for tracking clusters of people can be divided in three stages: detection of moving objects, data association, and join of clusters.

\section{B. Detection of moving objects}

This part of the system depends on the type of sensors. In our case, the sensors that have been used are laser range scanners. The existence of moving objects in the surrounding of the robot appears in the distance histograms of the lasers as local minima [9]. As other static obstacles (like the legs of a table, etc) can also have this characteristic, the set of local minima is filtered calculating the probability that the obstacle has moved. This is done estimating the difference between two consecutive occupancy grid maps. At the end of the process, the list of measurements that have been considered as moving objects is created: $Z(t)=\left\{z_{1}^{t}, \ldots, z_{n_{m}}^{t}\right\}$.

\section{Data association}

According to Eq. 1, the probability of a hypothesis can be calculated as:

$$
P\left(\Theta_{k}^{t} \mid Z^{t}\right)=P\left(\psi_{p}^{t}(k), \Omega_{p}^{t-1} \mid Z(t), Z^{t-1}\right)
$$

where $\psi_{p}^{t}(k)$ is a solution to the ambiguity matrix, $\Theta_{k}^{t}$ will be a hypothesis obtained after data association, and $\Omega_{p}^{t-1}$ is its parent hypothesis.

Applying Bayes's rule, and taking into account that

$$
\begin{gathered}
P\left(\psi_{p}^{t}(k) \mid \Omega_{p}^{t-1}, Z(t), Z^{t-1}\right)=P\left(\psi_{p}^{t}(k) \mid \Omega_{p}^{t-1}, Z(t)\right) \\
P\left(\Omega_{p}^{t-1} \mid Z(t), Z^{t-1}\right)=P\left(\Omega_{p}^{t-1} \mid Z^{t-1}\right)
\end{gathered}
$$

then Eq. 3 can be rewritten as ${ }^{1}$ :

$$
P\left(\Theta_{k}^{t} \mid Z^{t}\right)=P\left(\psi_{p}^{t}(k) \mid \Omega_{p}^{t-1}, Z(t)\right) P\left(\Omega_{p}^{t-1} \mid Z^{t-1}\right)
$$

${ }^{1} \Omega_{p}^{t-1}$ is the prior computed in the previous step of the Bayes filter. 
Thus, the probability of a hypothesis in the current iteration, will be the product of the probability of its parent hypothesis and the probability of an specific assignment of the measurements, i.e., a solution to the ambiguity matrix.

To model $P\left(\psi_{p}^{t}(k) \mid \Omega_{p}^{t-1}, Z(t)\right)$, all the possibilities for the assignments must be defined. These are:

- The measurement comes from a track inside a cluster, both belonging to $\Omega_{p}^{t-1}$. $P_{C T}^{c, f, m, t}$ will be the probability that track $f$ of cluster $c$ is assigned to measurement $m$ at time $t$.

- The measurement is generated by a new track inside a cluster that belongs to $\Omega_{p}^{t-1}$. $P_{C N}^{c, m, t}$ is the probability that cluster $c$ has a new track due to measurement $m$ at time $t$.

- The measurement is due to a new moving object that is not in any of the clusters in $\Omega_{p}^{t-1}$. The probability is defined as a constant, $P_{\text {new }}$.

- The measurement is noise, and the probability will also be modelled as constant, $P_{\text {noise }}$.

1) $P_{C T}$ : This probability is defined as:

$$
\begin{aligned}
P_{C T}^{c, f, m, t} & =P_{M}^{c, f, m, t} P_{D}^{c, f, t} \\
& =P_{M}^{c, f, m, t}\left(1-P_{\text {Nseen }}^{c, f, t}\right)\left(1-P_{\text {del }}^{c, f, t}\right)
\end{aligned}
$$

where $P_{M}^{c, f, m, t}$ is the probability that measurement $m$ belongs to track $f$, and it is calculated as:

$$
P_{M}^{c, f, m, t}=N\left[h\left(\bar{\mu}_{t}^{c, f}\right), H_{t}^{c, f} \bar{\Sigma}_{t}^{c, f}\left(H_{t}^{c, f}\right)^{T}+Q_{t}\right]
$$

being $N$ a normal distribution with mean equal to the measurement prediction, and with covariance matrix equal to the innovation covariance matrix associated to measurement $m$ and track $f$.

On the other hand, Eq. 7 also depends on the probability that a track is detected in the current iteration $\left(P_{D}^{c, f, t}\right)$. This is modelled with the probability of not seing the track (Eq. 9), and the probability of deleting the track from both the cluster and the hypothesis (Eq. 12):

$$
\begin{gathered}
P_{\text {Nseen }}^{c, f}=P\left(E_{\mathrm{occ}}^{c, f, t} \vee E_{\mathrm{out}}^{c, f, t}\right) \\
P\left(E_{\mathrm{occ}}^{c, f, t}\right)=\frac{\sum_{s=1}^{n s} P_{\text {occ }}\left(\bar{\mu}_{t}^{c, f, s}\right)}{n s} \\
P\left(E_{\mathrm{out}}^{c, f, t}\right)=\frac{\sum_{s=1}^{n s} P_{\text {out }}\left(\bar{\mu}_{t}^{c, f, s}\right)}{n s} \\
P_{d e l}^{c, f, t}=1-\exp \left\{\frac{-\sum_{i=t_{0}}^{t-1}\left(1-P_{\text {occ }}^{c, f, i}\right)}{\lambda_{d}}\right\}
\end{gathered}
$$

$P_{\text {Nseen }}^{c, f}$ is defined as the probability that the track is now occluded by one of the moving objects detected in the current iteration, or it is outside the scope of the robot. Both probabilities are estimated in the following way (Eqs. 10, 11): the probability density function of the track in the previous iteration is sampled, and the state of each sample $\left(\mu_{t-1}^{c, f}\right)$ is used for calculating a prediction of the state of the track. With this prediction, $\bar{\mu}_{t}^{c, f, s}$, the probability of occlusion (out of scope) is calculated. Finally the probability of occlusion (out of scope) of the track is estimated with the average of the probabilities of occlusion (out of scope) of the samples.

The probability of deletion of a track from a cluster, $P_{d e l}^{c, f, t}$, is modelled with an exponential to simulate the decay in the probability of detecting it when it is not seen in several consecutive iterations. $\lambda_{d}$ gives the speed of the decay process, while the summatory in the numerator of the exponential adds the probabilities that the track was not occluded along the time interval in which it was not detected $\left(t_{0}\right.$ to $\left.t-1\right)$.

2) $P_{C N}:$ The probability that the measurement comes from a new track of an existing cluster is defined as:

$$
P_{C N}^{c, m, t}=P_{b e l}^{c, m, t} P_{n e w_{\text {track }}}
$$

being $P_{n e w_{\text {track }}}$ a constant value representing the probability of appearance of a new track inside a cluster, and $P_{b e l}^{c, m, t}$, the probability that measurement $m$ belongs to cluster $c$ :

$$
P_{b e l}^{c, m, t}=P\left(\left\{\bigvee_{i=1}^{n f_{c}} E_{M}^{c, i, m, t}\right\} \vee E_{M}^{c, m, t}\right)
$$

The probability is the likelihood that the measurement belongs to one of the tracks in the cluster $\left(n f_{c}\right.$ is the number of tracks in cluster $c$ ), or that it belongs to the cluster.

3) Hypothesis probability: Recovering from Eq. 6, the first term of the right part can be rewritten as:

$$
\begin{aligned}
& P\left(\psi_{p}^{t}(k) \mid \Omega_{p}^{t-1}, Z(t)\right)=\left(P_{\text {new }}\right)^{n_{\text {new }}}\left(P_{\text {noise }}\right)^{n_{\text {noise }}} \\
& \left\{\prod _ { c = 1 } ^ { n c } \left\{\prod_{f=1}^{n f_{c}}\left\{P_{M}^{c, f, m_{c, f}, t}\left(1-P_{\text {Nseen }}^{c, f, t}\right)\left(1-P_{\text {del }}^{c, f, t}\right)\right\}^{\delta_{f}}\right.\right. \\
& \left.\left.\left\{1-\left(1-P_{\text {Nseen }}^{c, f, t}\right)\left(1-P_{d e l}^{c, f, t}\right)\right\}^{1-\delta_{f}}\right\} \prod_{g=1}^{n g_{c}} P_{C N}^{c, m_{c, g}, t}\right\}
\end{aligned}
$$

where $\left(P_{\text {new }}\right)^{n_{\text {new }}}\left(\left(P_{\text {noise }}\right)^{n_{\text {noise }}}\right)$ is the probability of assigning $n_{\text {new }}\left(n_{\text {noise }}\right)$ measurements as new moving objects (as noise). The product in $c$ (Eqs. 16, 17) represents the likelihood of the cluster. This can be divided in two products: the product in $f$ (Eqs. 16, 17), which evaluates the probability of the assignment of measurements to existing tracks in the cluster $\left(m_{c, f}\right.$ is the measurement assigned to track $f$ in cluster $c$ ), and the product in $g$ (Eq. 17), which represents the probability of new measurements inside the cluster $\left(n g_{c}\right.$ is the number of new measurements in cluster $c$ ).

Going into the details of the product in $f$, variable $\delta_{f}$ takes a value of one if for this assignment there is any measurement corresponding to track $f$, and zero otherwise. Thus, Eq. 16 represents the likelihood of the assignments of the measurements to the tracks, and Eq. 17 is the probability of not detecting a track. 
Once all the probabilities have been defined, the ambiguity matrix for each parent hypothesis can be constructed, and all the obtained matrices are then inserted in the Murty's algorithm, in order to obtain the $k$-best hypothesis, $\Theta^{t}$.

4) Split of clusters: After the data association, each cluster belonging to each of the obtained hypothesis, $\Theta_{k}^{t}$, must be examined for checking if its tracks have very different states. In that case, the cluster should be splitted in several new clusters that substitute the parent cluster in the hypothesis. The similarity of two different tracks, $(i, j)$, in a cluster is defined as:

$$
P_{s i m}^{i, j}=P\left(E_{\mu_{i}}^{\Sigma_{i}}\left(\mu_{j}\right) \vee E_{\mu_{j}}^{\Sigma_{j}}\left(\mu_{i}\right)\right)
$$

where $P\left(E_{\mu_{i}}^{\Sigma_{i}}\left(\mu_{j}\right)\right)$ is the probability of $\mu_{j}$ in the normal distribution with average $\mu_{i}$ and covariance $\Sigma_{i}$. Based on each $P_{s i m}^{i, j}$ a matrix of similarities is constructed for each cluster. If the cluster is splitted, the new clusters will be generated using the information of the similarities of the tracks, in order to keep homogeneous clusters.

\section{Clusters Join}

In the same way as for the previous stage (Eqs. 3, 6), the probability of a hypothesis is estimated as:

$$
P\left(\Omega_{k}^{t} \mid Z^{t}\right)=P\left(\phi_{p}^{t}(k), \Theta_{p}^{t} \mid Z^{t}\right)
$$

where $\phi_{p}^{t}(k)$ is a solution to the ambiguity matrix of clusters join, $\Omega_{k}^{t}$ is a hypothesis obtained after the clusters join stage, and $\Theta_{p}^{t}$ is its parent hypothesis, i.e., one of the hypothesis obtained after data association.

Applying Bayes's rule, and taking into account that

$$
P\left(\phi_{p}^{t}(k) \mid \Theta_{p}^{t}, Z^{t}\right)=P\left(\phi_{p}^{t}(k) \mid \Theta_{p}^{t}\right)
$$

then Eq. 19 can be rewritten as:

$$
P\left(\Omega_{k}^{t} \mid Z^{t}\right)=P\left(\phi_{p}^{t}(k) \mid \Theta_{p}^{t}\right) P\left(\Theta_{p}^{t} \mid Z^{t}\right)
$$

The simplification in Eq. 20 is valid as the ambiguity matrices for the clusters join stage, $\phi_{p}^{t}$, only depend on the state of the clusters of the hypothesis, and this information is already provided by $\Theta_{p}^{t}$. Thus, the probabilities we want to obtain are again the product of the parent hypothesis likelihood, and the probability of a solution to the ambiguity matrix.

The ambiguity matrices have two different types of assignments:

- A couple of clusters, $(i, j)$ can be joined. The probability will be $P_{j o i n}^{i, j}$.

- The cluster remains independent. This is modelled with a constant probability, $P_{\text {indep }}$.

$P_{j o i n}^{i, j}$ is defined in as:

$$
P_{j o i n}^{i, j}=N_{\mu_{i}}^{\Sigma_{i}}\left(\mu_{j}\right)
$$

Finally, Eq. 21 is rewritten as:

$$
P\left(\Omega_{k}^{t} \mid Z^{t}\right)=\left\{\prod_{c=1}^{n c}\left(P_{\text {join }}^{i, c}\right)^{\tau_{c}}\left(P_{\text {indep }}\right)^{1-\tau_{c}}\right\} P\left(\Theta_{p}^{t} \mid Z^{t}\right)
$$

where $\tau_{c}$ takes a value of one if the cluster joins another one, and zero otherwise.

1) Pruning: The described system has been implemented using three different trees for the hypothesis, clusters and tracks. In that way a track can be shared by several clusters, and a cluster can also be shared by several hypothesis. The hypothesis tree is pruned in each iteration of the algorithm in order to reduce the complexity using an "N-scan-back" algorithm [12]. This algorithm assumes that any ambiguity at time $t_{\text {now }}-$ tree $_{\text {depth }}$ can be solved at $t_{\text {now }}$. Thus, for each hypothesis at $t_{\text {now }}-$ tree $_{\text {depth }}$, the sum of probabilities of their offspring at $t_{\text {now }}$ is calculated, and the branch of the hypothesis with the highest sum is maintained, while the hypothesis of the other branches are pruned. Finally the cluster tree is pruned deleting those clusters that do not belong to any hypothesis, and also the ones that are empty (with no tracks). In the same way, the track tree is pruned taking into account the tracks that do not belong to any cluster, and also those tracks with a probability of deletion $\left(P_{d e l}^{c, f, t}\right)$ over a certain threshold, or that have been out of the scope of the robot for several iterations.

\section{EXPERIMENTAL RESULTS}

The experiment has been done with a Pioneer II robot equipped with two laser range scanners. The lasers were mounted at a height of $40 \mathrm{~cm}$ (front laser) and $60 \mathrm{~cm}$ (rear laser), and with a resolution of 0.5 degrees. Thus, one laser scan provides information of the whole surrounding of the robot. Due to this disposition of the lasers the legs of people will be detected. The experiment took place in the hall of a building of the University of Freiburg and lasted 9 minutes. During this time up to 10 people were tracked (that means 20 possible tracks at the same time). The size of the groups of people (clusters) ranged from 1 to 6 people, and the maximum number of clusters tracked at the same time was of 5. During the experiment, more than half of the time the robot was moving.

Fig. 1 shows part of the experiment, from cycle 177 to 240 . Both the laser scan and the best hypothesis are displayed in the most significant cycles of this part of the test. Several local minima in the distance histograms of the lasers appear in the lower part of all the laser scan figures. All of them are noise due to the presence of objects in the environment like legs of chairs or tables. Most of them are filtered calculating the difference between consecutive grid maps. The others can be filtered with the MHT algorithm, as it explicitly manages this possibility.

A detailed figure for the best hypothesis in a cycle is shown in Fig. 2. The static obstacles are represented with bars and the robot is represented as a black dot. The PDFs of the clusters are displayed by sets of ellipses. Each ellipse represents a normal distribution where the two radius of the ellipse are the standard deviations for the $x$ and $y$ variables of the state. The set of ellipses for each cluster corresponds to each one of its tracks, and also to its own state. The center of each track 


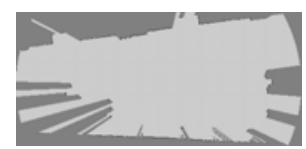

(a) Laser scan (177)

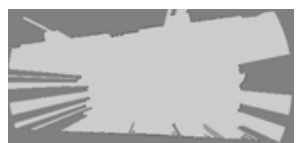

(b) Laser scan (182)

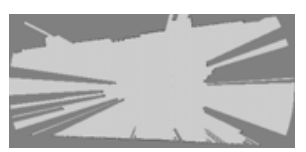

(c) Laser scan (191)

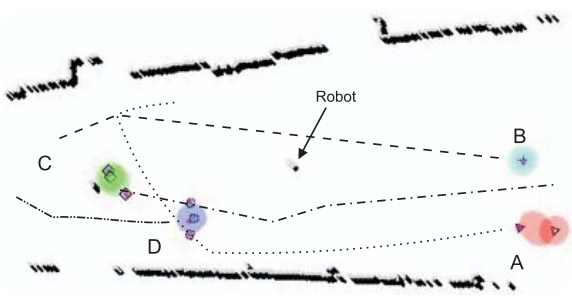

(d) Best hypothesis (177)

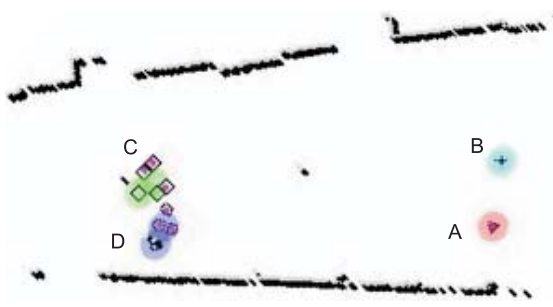

(e) Best hypothesis (182)

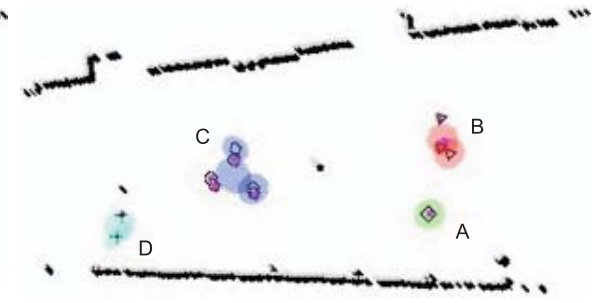

(f) Best hypothesis (191)

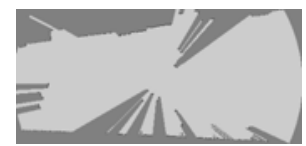

(g) Laser scan (200)

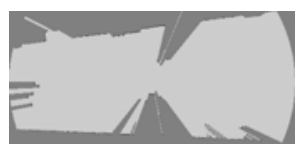

(h) Laser scan (203)

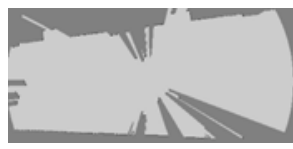

(i) Laser scan (207)

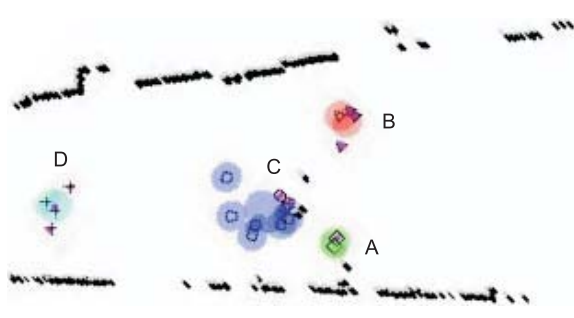

(j) Best hypothesis (200)

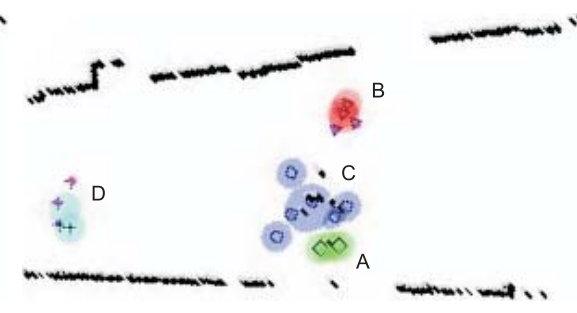

(k) Best hypothesis (203)

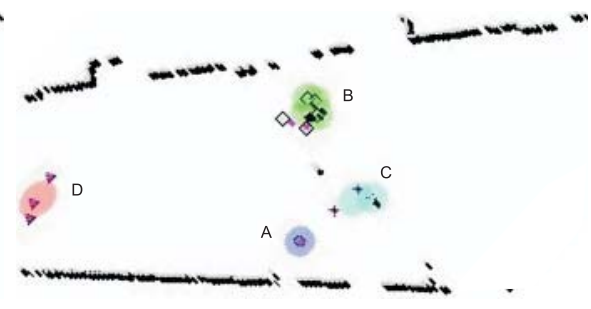

(1) Best hypothesis (207)

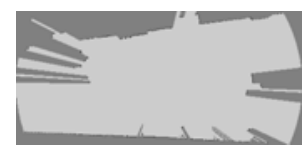

(m) Laser scan (227)

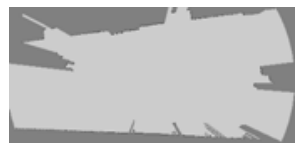

(n) Laser scan (232)

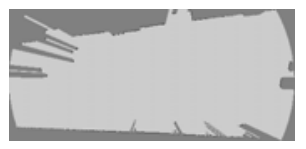

(o) Laser scan (240)

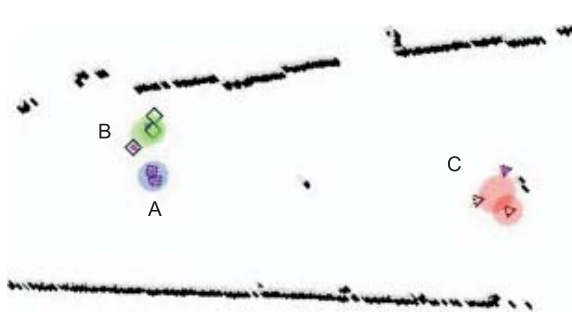

(p) Best hypothesis (227)

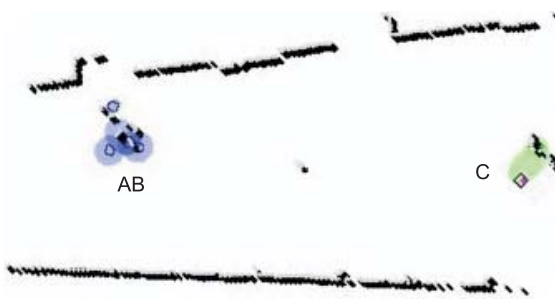

(q) Best hypothesis (232)

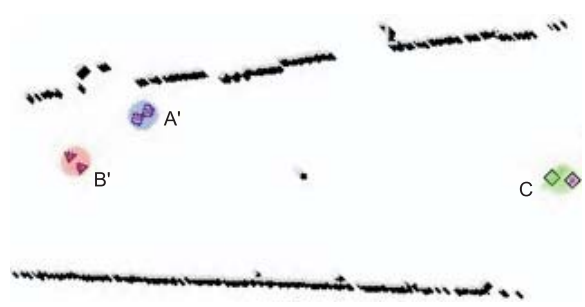

(r) Best hypothesis (240)

Fig. 1. Laser scan and best hypothesis in part of the experiment (cycles 177 to 240)

is also marked with a symbol (a triangle, circle, square or a cross), while measurements are represented by dots.

Fig. 1(d) shows the initial situation at the beginning of this part of the experiment. Cluster $A$ has one person and clusters $B-D$ three people each. The different traced lines represent the approximate trajectories that the clusters followed during the displayed sequence. As the number of people in a cluster increases, the occlusions between them make it virtually impossible to detect all the tracks of a group. For clusters $B-D$ the higher number of mobile objects detected along the displayed sequence was of four, while the real number was six. In fact, several times only one or two tracks are detected, as it happens, for example, with cluster $B$.

At cycle 182 (Fig. 1(e)), clusters $C$ and $D$ get very close, but there is no join of these clusters as the states are quite different due to their heading directions. From that cycle to cycle 196 , cluster $D$ is partially or totally occluded by cluster C. As an example, in cycle 191 (Fig. 1(f)) only one track is detected in cluster $D$ (the cluster keeps track of two), while four measurements are detected for cluster $C$ (the cluster keeps track of six). The same occurs with cluster $A$, that is occluded by cluster $C$ several cycles (Figs. 1(k) to 1(1)). 


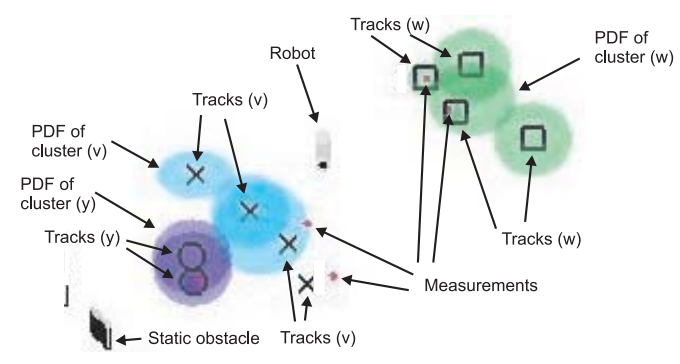

Fig. 2. Detail of a figure for the best hypothesis

At cycle 227 (Fig. 1(p)), cluster $A$ (one person) approaches to $B$ (three people), and the clusters join a few cycles later. The new cluster $A B$ has four people and, after a short time, it is splitted in two clusters moving in opposite directions, each one with two people.

In order to test the advantages of keeping track of clusters instead of individual tracks, the same experiment was repeated, but without the use of clusters. For both experiments, wrong data associations were manually counted. An erroneous data association for the cluster-based approach consists in assigning a measurement to a wrong cluster. On the other hand, for the track-based approach, an error consists in assigning a measurement to a wrong track. The explanation of this difference in the consideration of the errors is the following: a good tracking must be able to reconstruct the path followed by the tracked object. In the cluster-based approach, for example, a track can be initially in one cluster, afterwards split from that cluster and create a new one, and finally join another cluster. Its path can be reconstructed with the information about all the clusters it has belonged to, so the only source of error is an incorrect assignment of a measurement to a cluster. In the track-based approach, the reconstruction of the path is based on the measurements assigned to the track, so a wrong assignment or a deletion of the track can give rise to the impossibility of reconstructing that path.

Fig. 3 shows the comparison between both approaches. Of a total of 423 data associations in these cycles, the trackbased approach makes 75 errors $(17.73 \%)$ while the clusterbased approach has $2(0.47 \%)$. The improvement is clear, and enhances the usefulness of the cluster-based approach in those situations in which the objects to be tracked are quite close among them.

\section{Conclusions}

In this paper we presented a new MHT based approach for tracking clusters of people. Our method differs from existing tracking approaches (including those based in MHT) in that it tracks clusters of objects whenever they get close to each other instead of tracking all of them individually. The advantage of this approach becomes apparent in situations in which the objects that must be tracked have very similar states. In such situations, the data association problem gets much harder, not only because the observed features are close, but also because of the huge number of occlusions.

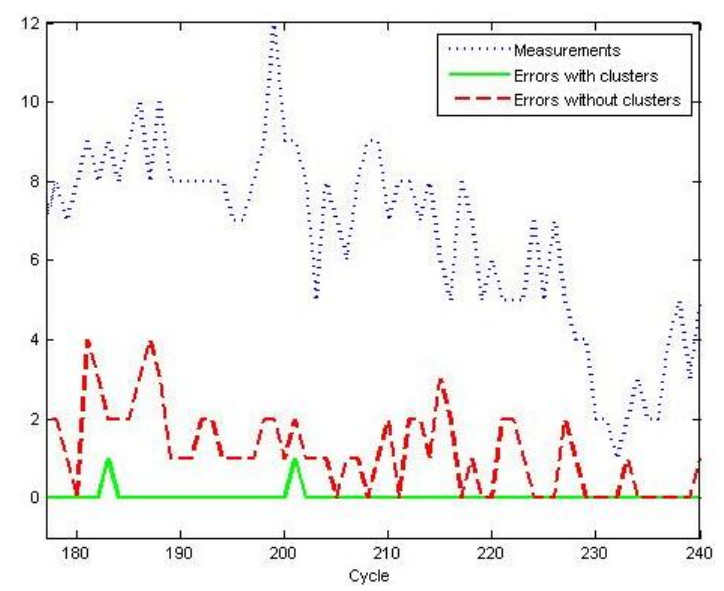

Fig. 3. Number of measurements and wrong data associations

Our algorithm has been implemented and tested on a Pioneer II robot equipped with two laser range scanners that cover the whole surrounding of the robot. Practical experiments, in which the robot had to track up to 10 people walking in several groups, demonstrate that our algorithm can reliably deal with such situations. Additionally, it has shown a performance that was superior to a standard MHT-based system that tracks all objects individually.

\section{ACKNOWLEDGMENT}

This work was done during a research stay of the first author at the University of Freiburg, supported by a grant of the Xunta de Galicia, It was also partially supported under grant PGIDIT04TIC206011PR of DXID of the Xunta de Galicia.

\section{REFERENCES}

[1] L. Taycher, J. Fisher, and T. Darrell, "Combining object and feature dynamics in probabilistic tracking," in Proc. Int. Conf. on Computer Vision and Pattern Recognition, 2005, pp. 106-113.

[2] M. Rosencrantz, G. Gordon, and S. Thrun, "Locating moving entities in indoor environments with teams of mobile robots," in Proc. 2nd Int Conf. on Autonom. Agents and Multiagent Syst., 2003, pp. 233 - 240.

[3] D. Reid, "An algorithm for tracking multiple targets," IEEE Transactions on Automatic Control, vol. AC-24, no. 6, pp. 843-854, 1979.

[4] I. Cox and S. Hingorani, "An efficient implementation of reid's multiple hypothesis tracking algorithm and its evaluation for the purpose of visual tracking," IEEE T. Patt. Anal. Mach. Intell., vol. 18, pp. 138 - 150, 1996.

[5] T. Schmitt, M. Beetz, R. Hanek, and S. Buck, "Watch their moves: Applying probabilistic multiple object tracking to autonomous robot soccer," in Proc. 18th Nat. Conf. on Artif. Intell., 2002, pp. 599-604.

[6] H. Tao, H. Sawhney, and R. Kumar, "A sampling algorithm for tracking multiple objects," in Workshop on Vision Algorithms, 1999, pp. 53-68.

[7] M. Isard and J. MacCormick, "Bramble: A bayesian multiple-blob tracker," in Proc. Int. Conf. Computer Vision, 2001, pp. 34-41.

[8] G. Taylor and L. Kleeman, "A multiple hypothesis walking person tracker with switched dynamic model," in Proc. Australasian Conf. on Robotics and Automation, Canberra (Australia), 2004.

[9] D. Schulz, W. Burgard, D. Fox, and A. Cremers, "People tracking with mobile robots using sample-based joint probabilistic data association filters.” Int. J. of Robotics Research, vol. 22, no. 2, pp. 99-116, 2003.

[10] K. Murty, "An algorithm for ranking all the assignments in order of increasing cost," Operations Research, vol. 16, pp. 682-687, 1968.

[11] I. Cox and M. Miller, "On finding ranked assignments with application to multitarget tracking and motion correspondence," IEEE Transactions on Aerospace and Electronic Systems, vol. 31, pp. 486-489, 1995.

[12] T. Kurien, Multitarget-Multisensor Tracking: Advanced Applications. Norwood, MA (USA): Artech House, 1990, ch. Issues in the design of practical multitarget tracking algorithms, pp. 43-83. 